\title{
Blockchain Use Cases and Their Feasibility
}

\author{
Kaspars Zīle ${ }^{1 *}$, Renāte Strazdiṇa ${ }^{2}$ \\ ${ }^{1,2}$ Riga Technical University, Riga, Latvia
}

\begin{abstract}
The goal of the paper is to provide a vague summary of currently existing blockchain use cases in the information technology industry. Respective use cases have been examined in already existing scientific papers, Master Theses, industry white papers and blogs of industry experts. The paper also contains a description of blockchain main technological aspects and working principles, which allows making the assessment of the presented use cases. For each use case respective companies or organisations are added that are applying or testing the given solution. Due to research limitations the paper should not be considered an exhaustive blockchain use case description.

The paper also provides short introduction into a feasibility analysis of specific blockchain use case. The authors describe the basic steps of potential idea evaluation with regards to blockchain main aspects. It helps understand the necessity for development of a detailed blockchain feasibility model.
\end{abstract}

Keywords - Bitcoin, blockchain, distributed ledger, feasibility, immutability, use case.

\section{INTRODUCTION}

In the information technology world, at least once in a few years a new technology emerges that seems to be an answer to all problems and the beginning of a new golden era. Since 2016, this technology has been blockchain, although the first version was already created in 2008 [1] and the basic idea was incepted by Haber and Stornetta already in 1991 [2]. The name is selfexplanatory, because blockchain consists of blocks, which are added one after another, just as in a famous game "Snake". From the functional point of view, it is a decentralised database without central authority, which uses TCP/IP protocol for communicating between its $\mathrm{P} 2 \mathrm{P}$ network members, or, in other words, it is a system that performs accurate and irreversible data transfer in a decentralised P2P network [2].

It is presumed that the first practical solution with the use of blockchain is cryptocurrency Bitcoin [3], [2]. Its value has been grown more than 60000 times since it has appeared, and it is one of the main reasons for blockchain current popularity. Unfortunately, it is also a somewhat negative factor, because very often blockchain is confused with Bitcoin, and most studies are focused only on Bitcoin's blockchain, although there can be countless variations and technical nuances. For example, it has been found out that the proportion of scientific papers about Bitcoin to the papers about other blockchain application possibilities is around 4:1. Overwhelming majority of research is focusing on revealing and improving limitations of blockchain from privacy and security perspectives, not exploring its possible applications out of value recording scope [4].
As many authors [5]-[7] indicate, currently blockchain is considered a breakthrough invention, which could change many everyday activities and business processes in different application domains, for example, to record election votes, ensure transparency in accounting, track property rights of luxury items, intellectual property rights, etc. However, as it usually happens with new technologies, there is a lot of hype around blockchain; therefore, expectations may exceed the reality [7]. All the buzz and hype has also created a large number of trend-followers, who are trying to present blockchain as a solution to all problems. Nevertheless, there are several technical challenges, such as scalability, integrity of network participants, distribution of computational power, reaching of consensus, preserving confidentiality of users and safety of the used encryption algorithms [8]-[10].

Another issue is a lack of clear understanding of blockchain uses and goals. Some authors consider that the main goal of blockchain technology is to create a decentralised environment where no third party is in control of the transactions and data [4]; some argue that it should facilitate cloud computing and immutable database usage [11], and others would like to use it mainly as a global software execution platform for smart contracts [12], [13]. Besides, there are numerous discussions and comparisons of advantages and drawbacks with public and private blockchains, where each type has specific features [14] and totally opposite application possibilities, security risks, etc. This all highlights the necessity for additional research and evaluation of different blockchain application options.

\section{BLOCKCHAIN IN THEORY AND PRACTICE}

\section{A. Related Work}

In addition to scientific papers and a few books, significant attention was given to articles and white papers written by blockchain enthusiasts and developers and published in different IT related internet resources. Blockchain itself is a very recent area of research and there is no extensive research conducted so far in the area. Most discussions and new discovery publications are taking place in different internet forums or blogs, e.g., an original paper of Bitcoin by Satoshi Nakamoto [1] published at his internet page was never reviewed as a proper scientific paper and did not even have a term "blockchain" in it. Nevertheless, today it is considered one of the most important papers in this area. There are a number of recent books about the blockchain, but most of them are trying to exploit popularity of cryptocurrencies and are nothing more than compilation of different Bitcoin descriptions. Only a few of them (e.g., Bitcoin and Cryptocurrency Technologies written

*Corresponding author's e-mail: kaspars.zile@outlook.com 
by Narayanan et al. and published by Princeton university in 2016 [2]) are serious scientific studies and can be used as a reference to further works.

The reviewed literature allows describing principles and foundation of blockchain technology, as well as its applications in different real-life use cases. It is worth mentioning that almost all papers indicate a need for further research in the field. A lot of authors believe that blockchain has significant potential, but still there are a lot of obstacles and challenges for real-life implementation. For example, there is not even single, worldwide accepted definition of blockchain; therefore, developing of common standards and regulations (both technical and legal) is highly necessary. Standardisation would be the first step towards formulating unified approaches in blockchain software development and security practices, without which a reasonable employment of blockchain is not feasible.

\section{B. Definition of Blockchain}

There are numerous blockchain definitions by different authors, and as pointed out in [15], there is no single, internationally agreed definition; therefore, it is important to understand the main parts of blockchain. Some have the opinion that blockchain technology has not been clearly defined yet [16]; therefore, they use Bitcoin as a reference point and use its three main parts - transactions, consensus, and network. Hileman \& Rauchs [3] offer definition, where distributed ledger is described as a type of distributed database that can have different users (nodes), and blockchain, in turn, is a type of distributed ledger, which is created like a chain of cryptographically linked 'blocks' with transactions and is sending all data to all nodes in its network. Tama et al. [17] are of similar opinion, describing blockchain as "part of the implementation layer of a distributed software system", whose purpose is to ensure data integrity. According to [18], the main difference between blockchain and regular database is that blockchain is the improved database with some sort of an automated solution for new record adding, validating and distributing the information over P2P network. Some authors neglect more detailed parts of blockchain and focus just on data integrity, for example, [19] states that blockchain is simply a cryptographically verifiable list of data.

Quite often the proposed definitions either include only a narrow spectrum of possible blockchain types or are limited to their description. One of the best ways how to define blockchain is by selecting features, without which the basic principles of blockchain would disappear. By doing this operation, it is possible to say that blockchain is a data structure with the following key elements [20]:

- data redundancy (each node has a copy of the blockchain);

- check of transaction requirements before validation;

- recording of transactions in sequentially ordered blocks, whose creation is ruled by a consensus algorithm;

- transactions based on public-key cryptography and a transaction scripting language.

Some authors [15], [21] would also add to such a list consensus mechanisms, which are meant to provide incentives for users to participate in a network, thus enabling greater overall computing power that leads to faster transaction processing and higher data security. An example to a consensus mechanism is Bitcoin's blockchain, where miners (network nodes) receive reward (coins) for processing transaction and creating blocks.

Debate about the definition of blockchain becomes especially important, when dealing with legal matters, such as passing laws regarding the use of blockchains. As Jeffries [22] points out, large differences in definitions may cause unpredictable problems in the future as states pass blockchain-related legislation. Currently some countries (Estonia, Switzerland, Arizona in the U.S.) have in place the basic legal framework for blockchain, which also contains its definition. Walch [23] and Stark [24] have made comprehensive research about blockchain definitions and law, where it is indicated that most subjects, who are passing blockchain-related legislation, mainly do it to demonstrate how crypto-friendly they are, but the quality of technology description is lagging.

In conclusion, it should be noted that blockchains can have a wide array of functionally different types [25]; therefore, one definition will not always be adaptable to all cases. However, in any description of blockchain its key idea should be included to store data and provide trust in their authenticity.

\section{Main Features of Blockchain}

As the name suggests, blockchain is a chain of blocks. Usually each block contains transactions and hash pointer, which serves as a link to the next block. In such a way, it is impossible to delete any block or insert a new one in the middle of a chain, because then hashes will not match. Blocks are created by network participants, who are processing transactions by running blockchain's client software. Such a participant is called a "node". To attach the compiled block of transactions to blockchain, a node must resolve a hash function of the block that satisfies certain mathematical conditions [26], although rules for block attaching can differ and depend on a respective consensus algorithm, which is used in a particular blockchain.

For a block to be included in a chain, a network consensus must be reached. It means that all network participants must confirm its authenticity. Depending on technical parameters, a block is included in a chain after some certain number of confirmations; however, it is being validated by all network nodes, until everyone has an up-to-date blockchain structure. Some studies [2] consider a proof-of-work consensus as the main innovation from Satoshi Nakamoto's blockchain, which is known as Bitcoin. Exactly this mechanism replaces the central authority and provides incentives that would keep members of the network honest.

Consensus can be reached in different ways, which depend on the technical approach used (proof-of-work, proof-of-stake, etc.). As blockchain is a distributed system, then consensus is also distributed [27], which means a higher degree of difficulty due to such factors as network latency, crashes and possible malicious network participants. In [2], it is mentioned that despite theoretical difficulties and high possibility of 
subversion, current applications of distributed consensus (Bitcoin, others) are working even better than they should, although there is not an exact, scientifically proven explanation for that.

One of the main principles of blockchain is immutability of the recorded entries. This is established as non-negotiable by practically all blockchain evangelists and enthusiasts, in whose opinion adding any degree of "editability" to blockchain defeats the very purpose of using it [15]. For this purpose, a wide range of other computational and mathematical tools are used, for example, Bitcoin uses hash pointers, which link the previous block to next one, and Merkle tree roots, which allow for the efficient whole blockchain validation [2]. Some blockchains involve certain parts of game theory (e.g., problem of Byzantine generals) for reaching consensus within a decentralised network, where there is a possibility of network subverting by malicious users. Exact mechanisms for achieving immutability depend on the intended application of blockchain and its parameters, such as scalability and accessibility [28].

Another important part of blockchain technology is data distribution. Again, it can be very different due to the previously described accessibility parameters. Blockchain can use classical data distribution models - peer-to-peer and client-server models [18], and each has consist advantages and drawbacks, which must be carefully assessed against the intended goals. For example, peer-to-peer data distributing will be less reliable, but it will offer greater decentralisation and, therefore, better protection against closing the network. Distributed systems can also achieve greater computational power by combining it from users, but it comes with cost of possible damage done by malicious users [17].

\section{Main Risks of Blockchain}

Possibilities always come along with risks. Each core function of blockchain has several significant threats that need to be evaluated and counter measured before implementation. Not always these risks will be purely technical, because risks can also arise from legal, economical, even cultural areas.

One of the main concerns regarding decentralised networks is always about their control (see also [29]). For example, blockchains with a proof-of-work consensus algorithm are theoretically vulnerable to the so-called 51-percent attack, when one network node would have $51 \%$ of total network computational power, thus gaining possibility to singlehandedly confirm transactions and create new blocks [2]. However, to implement such an attack, vast computational power would be necessary, which eradicates the economic feasibility of such an act. Hofmann et al. [30] have calculated that as of October 2017 for such an attack it would be necessary to generate more than 5 million TH/s, which would cost around EUR 470 million. Similar possible threat is Sybil attack, where a malicious node floods a network with "fake" users that are controlled by it [19], but in this case employing a proof-of-work consensus actually helps mitigate the risk of such an attack, because nodes cannot modify a network without having computational power (see also [31]). Therefore, in case of public blockchains we can be relatively sure about their network safety, once there is an adequate number of network users that cannot be easily subdued.

Not always immutability is a desired feature. This may be useful for irreversible proof ensuring, but not in case of important financial transactions. The most famous example in blockchain history is the so-called "DAO wars". DAO stands for "Decentralised Autonomous Organisation", and it was one of the first Ethereum-based blockchains. In 2016, somebody found vulnerability and used it to transfer DAO tokens to himself from other users in a total amount of EUR 50 million [30]. Afterwards, Ethereum blockchain was forked (split in different branches) due to a majority of network participants not willing to continue to support blockchain with happened theft. However, that part of Ethereum blockchain still continues to function and is known as Ethereum Classic. And even in cases, when immutability is exactly what is needed, there always are risks connected to record reliability [32], which must be mitigated by proper network control and strict enforcement of consensus rules.

Considering the recent appearance of blockchain technology, one of the possible risks is still not very relevant, but with aging of the currently created blockchains it will become more important. It is long-time digital preservation [15], which in case of blockchains is not as straightforward as in usual centralised databases (see also [33]).

Another reason for possible security risks is a lack of academic research and subsequent standardisation of industry security measures. As Halpin \& Piekarska [19] point out, the current blockchain development is mainly done by practitioners without involvement of cryptography specialists; therefore, resistance and security solutions are ever-changing and different from case to case due to individual choices made by developers, which are based on their practical experience. It can also include the choice of defective protocols and implementation errors, such as unsecure scripting language design [34]. A lack of common vocabulary also creates additional problems in designing secure solutions, although there are attempts to create it [35].

Last, but not the least, there are risks emanating from the use of cryptography. Although the used algorithms, such as ECC and RSA, are generally regarded as safe, there is still a possibility to discover unknown vulnerabilities or backdoors, as it was a case with SHA-1 hashing algorithm [36]. Speculation about quantum computers and their alleged possibilities to easily decipher existing algorithms cannot be viewed as proper risk; nevertheless, it has a potential to materialise sometime in future. Use of private/public keys also implies risk from user's perspective - access denial to data due to losing a private key, which cannot be recovered or recalculated [4].

\section{BLOCKCHAIN APPLICATION IN DIFFERENT AREAS}

During 2016 and 2017, blockchain started to gain recognition in wider audiences, which led to a significant increase in the proposed services and software applications, which would be based on blockchain. Unfortunately, as it is common at an early adoption stage, there are a lot of unrealistic proposals and expectations, sometimes even deliberate fraud to trick the 
$2018 / 23$

investors. That is why it is very important to clearly understand limitations of blockchain, its possible applications and the following benefits.

It should come as no surprise that most numerous attempts of using blockchain are happening in the financial sector. In large part, this is due to Bitcoin and other cryptocurrencies, which have showcased the blockchain to the rest of the world. Hileman \& Rauchs [3] have estimated that about $30 \%$ of blockchain use cases are related to banking and financial services (see also [37]). It has also gained some traction in other sectors, such as government (13\%), insurance (12\%) and healthcare ( $8 \%$, see also [38]). However, as several authors indicate [39], [40], a blockchain technology is still at the initial stage of adaptation cycle and the expectations currently are very inflated. It is worth mentioning specifics of each individual sector, for example, Lamberti [41] indicates that insurance market characteristics (e.g., services offered, processes implemented, customers served, etc.) give sufficient reasons to doubt worthiness of blockchain adoption at all, because it might not be worth the investments due to a large number of related systems and their necessary adaptation.

Table I below presents a summary of some known software applications and use cases, where blockchain has been used as one of the main components (Table I, edited and compiled from the following sources: [2], [3], [8], [12], [13], [18], [42]-[51]). List is not to be considered complete, because it is not possible to gather information about all existing use cases. Due to rapid blockchain development, new solutions are also appearing daily. The table below is intended to give an overview and general idea about the most popular solutions that are used at present. Some of them are still only at the testing stage; thus, full functionality is not present. Classification of use cases into categories is only for easier overview and is not to be considered as official classification. Some of blockchain researchers would argue that all activities related to blockchain are data recording and immutable storage [16]; therefore, more detailed grouping is unnecessary, but it makes harder to perceive relevance of each use case.

TABLE I

BLOCKCHAIN USE CASES

\begin{tabular}{|c|c|c|}
\hline Category & Use case & Applier \\
\hline \multirow{10}{*}{$\begin{array}{c}\text { Data } \\
\text { management }\end{array}$} & Network infrastructure & $\begin{array}{l}\text { Eris, Mastercoin, } \\
\text { Chromaway, Nxt }\end{array}$ \\
\hline & $\begin{array}{c}\text { Content and resource } \\
\text { distribution }\end{array}$ & Swarm \\
\hline & Cloud storage & $\begin{array}{l}\text { Storj, Maidsafe, } \\
\text { PeerNova }\end{array}$ \\
\hline & Data monitoring & Modum.io \\
\hline & Identity data management & $\begin{array}{c}\text { UniquID, SolidX, } \\
\text { OneName, Trustatom, } \\
\text { uPort Microsoft, IBM, } \\
\text { ShoCard }\end{array}$ \\
\hline & Contract management & $\begin{array}{c}\text { Ethereum, Mirror, } \\
\text { Ottonomos, Symbiont }\end{array}$ \\
\hline & $\begin{array}{c}\text { Inter-organisational data } \\
\text { management }\end{array}$ & Multichain \\
\hline & $\begin{array}{l}\text { Tamper-proof event log } \\
\text { and audit trail }\end{array}$ & $\begin{array}{l}\text { Chronicled, Factom, } \\
\text { Securechain }\end{array}$ \\
\hline & System metadata storage & Blockstack \\
\hline & $\begin{array}{l}\text { Data replication and } \\
\text { protection from deleting }\end{array}$ & Securechain \\
\hline
\end{tabular}

\begin{tabular}{|c|c|c|}
\hline & $\begin{array}{l}\text { Digital content publishing } \\
\text { and selling }\end{array}$ & Alexandria.io, Ascribe \\
\hline & IoT sensor data purchasing & $\begin{array}{l}\text { DataBrokerDAO, } \\
\text { Chimera, Filament }\end{array}$ \\
\hline \multirow{7}{*}{ Data verification } & Photo \& video proofing & Uproov \\
\hline & Document notarisation & $\begin{array}{l}\text { BitCourt, Blocksign, } \\
\text { Enigio Time, Stampery }\end{array}$ \\
\hline & Work history verification & APPII \\
\hline & Academical certification & Sony Global Education \\
\hline & $\begin{array}{c}\text { Identity verification and } \\
\text { key management }\end{array}$ & $\begin{array}{l}\text { Microsoft, } \\
\text { Authentichain, } \\
\text { Everpass }\end{array}$ \\
\hline & $\begin{array}{c}\text { Product quality } \\
\text { verification }\end{array}$ & $\begin{array}{l}\text { Everledger, Verisart, } \\
\text { Bitshares, Bitreserve }\end{array}$ \\
\hline & Proof of origin & $\begin{array}{c}\text { Provenance, Tierion, } \\
\text { ArtPlus, Stampery }\end{array}$ \\
\hline \multirow{9}{*}{ Financial } & Trade finance & $\begin{array}{c}\text { Barclays, Santander, } \\
\text { BNP Paribas }\end{array}$ \\
\hline & $\begin{array}{l}\text { Currency exchange \& } \\
\text { remittance }\end{array}$ & $\begin{array}{l}\text { Kraken, Bitstamp, } \\
\text { Coinbase, BitPesa, } \\
\text { Bitso, Coincheck, } \\
\text { RobinHood, Huobi }\end{array}$ \\
\hline & $\mathrm{P} 2 \mathrm{P}$ payments & $\begin{array}{l}\text { Codius, BitBond, } \\
\text { BitnPlay, BTCjam }\end{array}$ \\
\hline & Crowdfunding & Waves, Starbase \\
\hline & Insurance & Insurechain \\
\hline & $\begin{array}{l}\text { Stock share and bond } \\
\text { issuing }\end{array}$ & Chain \\
\hline & $\begin{array}{l}\text { Central bank money } \\
\text { issuing }\end{array}$ & $\begin{array}{l}\text { Sweden, Russia (on } \\
\text { idea level) }\end{array}$ \\
\hline & Supply chain management & Eaterra, Profeth. \\
\hline & Value transfer and lending & $\begin{array}{c}\text { Ripple, Monero, } \\
\text { Bitcoin, Litecoin, } \\
\text { Zcash, etc. } \\
\end{array}$ \\
\hline \multirow{20}{*}{ Other } & Prediction recording & Augur, Gnosis \\
\hline & Social voting system & ThanksCoin \\
\hline & Ridesharing & Arcade City, La'Zooz \\
\hline & Domain name registration & Namecoin \\
\hline & Healthcare record storing & $\begin{array}{l}\text { DNA.bits, Medicare, } \\
\text { BitHealth, MedVault }\end{array}$ \\
\hline & $\begin{array}{c}\text { Software license } \\
\text { validation }\end{array}$ & IBM \\
\hline & $\begin{array}{l}\text { Content or product } \\
\text { timestamping }\end{array}$ & Po.et, Nexus Group \\
\hline & Lottery & Lastis, EtherPot \\
\hline & Property right registration & $\begin{array}{c}\text { Georgia Land Register, } \\
\text { Ascribe, ChromaWay, } \\
\text { BitLand }\end{array}$ \\
\hline & $\begin{array}{c}\text { Social rating } \\
\text { creation/monitoring }\end{array}$ & SOMA \\
\hline & Voting in elections & $\begin{array}{c}\text { European Parliament, } \\
\text { Ballotchain } \\
\end{array}$ \\
\hline & Marriage registration & Borderless.tech \\
\hline & Court proceedings & PrecedentCoin \\
\hline & Donations & BitGive \\
\hline & $\begin{array}{c}\text { Computational power } \\
\text { outsourcing for scientific } \\
\text { purposes }\end{array}$ & $\begin{array}{l}\text { SETI@home, } \\
\text { Folding@home }\end{array}$ \\
\hline & Electronic locks & Slock.it \\
\hline & Electro energy selling & TransActive Grid \\
\hline & Product tracing & Blockverify \\
\hline & Gaming & PlayCoin, Deckbound \\
\hline & Reviews \& endorsement & $\begin{array}{c}\text { TRST.im, Asimov, The } \\
\text { World Table }\end{array}$ \\
\hline
\end{tabular}

\section{CURRENT RESEARCH IN EVALUATION OF BLOCKCHAIN}

When considering different blockchain applications and use cases, it is necessary to perform at least a basic analysis of the proposed solution feasibility, because quite often an article or even a scientific paper proposes just a theoretical description 
without a clear idea of physical implementation. Quite many start-ups are also focusing mainly on attracting investors rather than creating useful and innovative solutions. A prominent example of such behavior can be initial coin offerings (ICO), which gained vast popularity in 2017 and were often viewed as scams by serious investors. There have been recent attempts (at the beginning of 2018) to establish some "guidelines" for evaluating ICOs, but these are mainly focusing on how to detect obvious scams [52]. It can be expected that growing popularity of ICOs will facilitate more research in this area and lead to appearance of rigid evaluation models.

In 2017, several researchers already made the first steps towards creating evaluation models for blockchain [9], [53] [57]. Depending on the depth of research and the intended goal, such models can be either very detailed or very simple, latter just helping quickly gain a general understanding of high-level feasibility of the idea. According to Lewis R. [48], if there are issues or problems with trust, consensus, immutability or a mix of the three within use case, then application of blockchain would be feasible, otherwise there is no real reason to apply it. In practice, these considerations could be easily applied in the first step of evaluation, thus helping understand if there is a necessity for further research.

Most scientific papers are dedicated to considering blockchain application possibilities within some certain area, for example, Smith [57] proposes three criteria that are a prerequisite for blockchain-based data management projects: dependability, security and trust. However, to fulfill these criteria, a project needs to satisfy additional parameters:

- the content of the blockchain must be dependably available to users;

- the blockchain and related applications must be secure;

- the blockchain and related applications and procedures must be trustworthy.

Smith [57] also used these criteria to perform basic evaluation test of several actual incepted projects (MedRec: Blockchain for Medical Records, Storj (Metadisk): Blockchain for Distributed Cloud Storage). However, these criteria practically do not differ from the widely known CIA principle in IT security management - confidentiality, integrity, availability - therefore, contribution of Smith is not groundbreaking.

Chinese researchers [54] state that often the main difficulty in assessing blockchain suitability for different use cases is a lack of clear product data (parameters). There is no further explanation, why these data cannot be obtained, but in some cases such a problem is present; however, it usually disappears in later development process, when detailed technical parameters are presented. Based on the existing blockchain product descriptions, technical forums, academic literature and their own experience of developing blockchain prototypes, they created multi-level framework [54], which can be used for initial blockchain suitability evaluation (see also [58]). This framework allows going through various technical aspects of blockchain and step-by-step arriving to a decision of using it or sticking to a conventional database. Further in work this framework was applied to four generic use cases (supply chain, electronic health records, identity data management and stock market), and, as a result, blockchain was deemed as suitable for using it in supply chain and identity data management projects. As the main deal-breaker for electronic health record case was data privacy, but for stock markets it was low performance. In general, this framework is quite simplified and predetermined; not giving many possibilities for variations, but nevertheless it allows performing adequate initial evaluation.

Similar model was created by Peck [55], although with different evaluation aspects. Peck provides three end options, discerning between public and private blockchain. Decision points (questions) are not so technically oriented, but more focusing on functional aspects of blockchain, especially trust. Peck also cites Gideon Greenspan, the CEO of Coin Sciences, who states that "if you don't mind putting someone in charge of a database, then there's no point using a blockchain", therefore indicating that matter of trust and its possible problem solutions are almost single justification for using blockchain.

More detailed research was performed by Canadian researcher Victoria Lemieux [15], [47], [53], who focused on possible blockchain application in archival preservation and recordkeeping. The main takeaway from her research was that blockchain record-keeping could bring a lot of benefits (increase transparency, protect privacy, improve efficiency), but there still was a large gap between the desired and existing solutions. The main problem is a lack of risk evaluation, and Lemieux even indicates that in some cases blockchain enthusiasts choose to omit any risk analysis due to fear of slowing possible innovation. Her research states that a lack of risk evaluation and analysis is directly related to overhyping blockchain as a technology and focusing only on its possibilities.

Regarding record-keeping and possible blockchain application for this purpose, Lemieux points out that most of blockchain innovators and developers are unaware of existing theories, principles, practices and standards for record-keeping. It was also discovered that some of presented solutions are misleading, for example, claiming to store data records on the blockchain, but in reality keeping only hash values of respective records [15]. To illustrate possible evaluation of blockchain suitability for record-keeping, Lemieux uses framework (see also [59]) from archival science, which determines data record as trustworthy if it is accurate, reliable and authentic. There is a detailed explanation of framework levels and composition, followed by applying it to a generic blockchain solution (meaning that no specific use cases were evaluated). In the evaluation process, several gaps are identified (incorrect transaction linking, possible inconsistencies due to timestamping and network latency) and possible solutions offered (creating an archival bond between data records by using transaction metadata or semantic layer between records).

Another detailed blockchain evaluation model was created by Japan's Ministry of Economy, Trade and Industry in cooperation with Mitsubishi Research Centre [60]. In the course of researching domestic/global surveys of literature and actual use cases of blockchain based systems, it has been established that an evaluation form covers both private and public 
blockchain solutions. Some parts were borrowed from ISO standards, for example, quality evaluation items were adjusted from ISO/IEC25010 (system and software quality model). Form was divided into three modules (quality, maintenance/operation, cost) and each module was divided into categories, which had several evaluation items, for example, in module Quality category Performance Efficiency had such items as throughput, network latency, block confirmation and data reference. There were also additional remarks regarding each category and evaluation item.

As indicated in description, this model is intended for system vendors who propose replacing existing systems with blockchain-based system to their clients and for evaluation of test results of blockchain-based systems. At the moment of publishing (April 2017), this model was not yet widely applied, and a key challenge was implementation of evaluation forms to actual systems and accumulation of evaluation cases [9].

Some researchers aim to evaluate different blockchain projects from technical aspects [61], [62]. Gencer \& Sirer [63] have created project Miniature World that provides a possibility to emulate an actual blockchain in a virtual environment and test it in different scenarios. For this purpose, they have developed specific metrics, such as mining power utilisation, fairness, consensus delay, time to win, and time to prune. However, such evaluation could be part of already later project stage, as it would not help determine whether the use of blockchain was necessary at all.

Similar approach was employed by Zhang et al. [10], who developed metrics for evaluating blockchain-based decentralised applications in healthcare. They created in total 7 metrics, which were ranked in order of significance, and detailed explanation of each metric was provided. It is worth noting that this evaluation model uses not simple, closed questions, but sets certain criteria, against which case must be analysed and evaluated. However, this evaluation model can be used only for a specifically described purpose (due to healthcare domain-specific technical requirements), and not for a wide range of initial assessment. Such a model also requires both familiarity with technical standards of a respective domain and experience with business processes. The drawback of the described research is a lack of model validation.

There are also a lot of sceptics, who (some with more, some with less argumentation) declare that blockchain is quite useless. Omitting Wall Street traders and investor celebrities, one of the most popular negative opinions about blockchain belongs to American entrepreneur Kai Stinchcombe [64], who published his analysis on Medium at the end of 2017. It states that during almost a decade since the first appearance of blockchain, there is still no any single working software solution. In his article, Stinchcombe goes through the main use cases of blockchain (smart contracts, micropayments, data distribution and storing, authenticity verification) and provides arguments against each case's feasibility (OR against feasibility of each case). Data storage is especially undesirable, as he argues that blockchain provides too much overhead by doing unnecessary work and omitting useful additional features. Additional problem is blockchain's single point of entry, i.e., usually for user to access his data, there is a need for password, and if it's forgotten or lost, then so are data. In Stinchcombe's opinion, for data or object verification distributed and encrypted ledger does not add any value - the same can be done by verifying the included certificate online.

His conclusion is that blockchain enthusiasts are too little interested in existing business processes and their added value. It leads to "reinventing the wheel" or even worse, creating products without any superior value to user than existing ones.

Others, for example, Ovenden [65] points out that current hype is one of the main factors that does not allow technology to develop at reasonable pace. Large tech companies such as IBM and Microsoft are being criticised for trying to create commercialised, centralised blockchain products, which go against initial blockchain ideology. Expectations of investors are much higher than possibilities of the created solutions; therefore, some sort of crash is imminent. In Ovenden's opinion, it would be even beneficial for blockchain, because it would allow for better identification of real use cases and provide time for creating legal regulation.

A lot of academics are quite sceptical about possible use of blockchain, e.g., Jorge Stolfi from the State University of Campina (Brazil), who even has earned nickname "professional Bitcoin troll" amongst supporters of Bitcoin. He dissected NIST report 8202 [66] and indicated multiple flaws of technology [67]. His main reasons against blockchain are the following:

- decentralisation is costly and not really necessary;

- only use for permissionless blockchains is cryptocurrency (because without any rewarding incentives network members would not act honestly);

- initial assumptions about members of permisionless blockchains as being "self-greedy" are not working in real life (shown with examples of Bitcoin reversals after major hackings and current centralisation of mining power).

In Stolfi's opinion, a majority of blockchain projects are conceived by enthusiastic but unqualified people, who have little or no experience in databases, distributed services, networking and financial computing. Their main error is assuming as an axiom that a blockchain technology is revolutionary and the currently existing technology is not relevant. In his usual sarcastic manner, Stolfi concludes his comment with suggestion to replace NIST report with a simple sentence - "Blockchains are useless".

Another recognised blockchain critic is David Gerard, who wrote pop-science book "Attack of the 50 Foot Blockchain" [68] in 2017, which was widely reviewed and discussed in popular culture. It is a non-technical review of the blockchain, Bitcoin and other cryptocurrencies, and quite detailed discussion of the technological potential. Although Gerard does not dismiss blockchain altogether, he is fairly sceptical of its hype, and proves that in many cases it does not offer any benefits and sometimes even creates more problems. His main point is that currently blockchain adds frictions to an already seamless process, for example, in payments. Existing digital payment providers and processors are ensuring much more user-friendly, faster and safer process then cryptocurrencies and blockchain systems. The main selling point of blockchain - 
decentralisation - does not really have a lot of advantages in most businesses; therefore, it has no incentives to offer for users [68]. He also describes various scams that have happened in blockchain landscape (and it is not a small number) and notes that it is one of the reasons for popularity of Bitcoin and other cryptocurrencies, because cybercriminals have vast opportunities to realise their ploys in this environment.

\section{CONCLUSION}

The present paper has provided an overview of the majority of currently existing and known use cases with blockchain technology application. Use cases are compiled from different sources, such as scientific papers, industry expert blogs, Master Theses and research. Scope of this paper is limited; therefore, a list of the mentioned use cases should not be considered exhaustive.

From an overview of use cases it is clear that a majority of blockchain applications relate to data management and data verification. These applications are mostly developed and used within the financial sector. The main reasons are blockchain introduction as cryptocurrency (Bitcoin), vast resources of large financial organisations and fast-paced innovative culture of these organisations. Other sectors, such as governmental institutions, are less flexible and therefore adaptation of blockchain is significantly slower.

The paper has described the basics of blockchain application feasibility evaluation. It can be done by examining the main components of blockchain functionality and finding out the correlation with a specified problem. It is worth mentioning that research in blockchain evaluation model is still only at a very early stage, and further research in this area is necessary. Correct evaluation of blockchain necessity can save a large number of resources in software development and maintenance costs.

\section{REFERENCES}

[1] S. Nakamoto, "Bitcoin: A Peer-to-Peer Electronic Cash System," 2008. [Online]. Available: https://bitcoin.org/bitcoin.pdf_[Accessed: 8 May 2018].

[2] A. Narayanan, J. Bonneau, E. Felten, A. Miller, and S. Goldfeder, Bitcoin and Cryptocurrency Technologies. Princeton University Press, 2016, $308 \mathrm{p}$.

[3] G. Hileman \& M. Rauchs, Global Blockchain Benchmarking Study. Cambridge, United Kingdom: Cambridge Centre of Alternative Finance, Sept. 2017. Available: https://ssrn.com/abstract=3040224 [Accessed: 8 May 2018]

[4] D. Yang, J. Gavigan, and Z. Wilcox-O'Hearn, Survey of Confidentiality and Privacy Preserving Technologies for Blockchains, 2016.

Available: https://www.r3.com/wp-content/uploads/2017/06/survey_ confidentiality_privacy_R3.pdf [Accessed: 8 May 2018].

[5] Citi Group. [Online]. Citi GPS: How FinTech is Forcing Banking to a Tipping Point, March 2016. Available: https://www.citivelocity.com/citigps/_[Accessed: 3 May 2018]

[6] Everis NEXT, "17 Blockchain Disruptive Use Cases," 31 May 2016. [Online]. Available:

https://everisnext.com/2016/05/31/blockchain-disruptive-use-cases/ [Accessed: 8 May 2018].

[7] R. Krawiec, D. Housman, M. White, M. Filipova, F. Quarre, D. Barr, A. Nesbitt, K. Fedosova, J. Killmeyer, A. Israel, and L. Tsa, Blockchain: Opportunities for Health Care. NIST Workshop on Blockchain \& Healthcare, Aug. 2016. Available: https://www2.deloitte.com/content/ dam/Deloitte/us/Documents/public-sector/us-blockchain-opportunitiesfor-health-care.pdf_[Accessed: 8 May 2018].
[8] M. Conoscenti, A. Vetro, and J. C. De Martin, "Blockchain for the Internet of Things: A Systematic Literature Review," in IEEE/ACS 13th International Conference of Computer Systems and Applications, 2016, pp. 1-6. https://doi.org/10.1109/AICCSA.2016.7945805

[9] "Survey on Establishing Evaluation Model for Blockchain," Mitsubishi Research Institute, 2017. Available: http://www.meti.go.jp/meti_lib/report/H28FY/000346.pdf [Accessed: 8 May 2018].

[10] P. Zhang, D. C. Schmidt, J. White, and G. Lenz, "Metrics for Assessing Blockchain-Based Healthcare Decentralized Apps," in IEEE 19th International Conference on e-Health Networking, Applications and Services, 2017 pp. 17-20. https://doi.org/10.1109/HealthCom.2017.8210842

[11] Z. Zheng, S. Xie, H. Dai, X. Chen, and H. Wang, "An Overview of Blockchain Technology: Architecture, Consensus, and Future Trends," in IEEE 6th International Congress on Big Data, June 2017, pp. 557-564. https://doi.org/10.1109/BigDataCongress.2017.85

[12] N. Bauerle, "What are the Applications and Use Cases of Blockchain?". [Online]. Available: https://www.coindesk.com/information/applicationsuse-cases-blockchains [Accessed: 8 May 2018]

[13] K. Christidis and M. Devetsikioti, "Blockchains and Smart Contracts for the Internet of Things," IEEE Access, vol. 4, pp. 2292-2303, 2016. https://doi.org/10.1109/access.2016.2566339

[14] BitFury Group and J. Garzik, "Public versus Private Blockchains Part1: Permissioned Blockchains, Part2: Permissionless Blockchains, 2015. Available: http://bitfury.com/docs/

[15] V. Lemieux, Blockchain Technology for Record Keeping: Help or Hype?, vol 1. University of British Columbia, 2016. Available: https://www.researchgate.net/profile/Victoria_Lemieux [Accessed: 8 May 2018].

[16] H. Okada, S. Yamasaki, and V. Bracamonte, "Proposed Classification of Blockchains Based on Authority and Incentive Dimensions," in 19th International Conference on Advanced Communication Technology, 2017. https://doi.org/10.23919/ICACT.2017.7890159

[17] B. A. Tama, B. J. Kweka, Y. Park, and K. H. Rhee, "A Critical Review of Blockchain and Its Current Applications," in International Conference on Electrical Engineering and Computer Science, 2017, pp. 109-113. https://doi.org/10.1109/ICECOS.2017.8167115

[18] A. Lewis, "So You Want to Use a Blockchain for That?," Jul. 2016. [Online]. Available: https://www.coindesk.com/want-use-blockchain/ [Accessed: 8 May 2018].

[19] H. Halpin and M. Piekarska, "Introduction to Security and Privacy on the Blockchain," in 2nd IEEE European Symposium on Security and Privacy Workshops, 2017. https://doi.org/10.1109/EuroSPW.2017.43

[20] S. Porru, A. Pinna, M. Marchesi, and R. Tonelli, "Blockchain-Oriented Software Engineering: Challenges and New Directions," in IEEE 39th International Conference on Software Engineering Companion, May 2017, pp. 169-171. https://doi.org/10.1109/ICSE-C.2017.142

[21] T. Swanson, Consensus-as-a-Service: A Brief Report on the Emergence of Permissioned, Distributed Ledger Systems. R3 CEV, 2015.

[22] A. Jeffries, "Blockchain” is Meaningless," The Verge, March 2018. [Online]. Available: https://www.theverge.com/2018/3/7/17091766/ blockchain-bitcoin-ethereum-cryptocurrency-meaning_[Accessed: 8 May 2018].

[23] A. Walch, "The Path of the Blockchain Lexicon (and the Law)," 36 Review of Banking \& Financial Law 713, 2017. Available: https://ssrn.com/abstract=2940335 [Accessed: 8 May 2018].

[24] J. Stark, Applications of Distributed Ledger Technology to Regulatory and Compliance Processes. R3, 2017. Available: https://www.r3.com/ wp-content/uploads/2017/07/apps-reg-compliance_R3.pdf_[Accessed: 8 May 2018].

[25] V. Buterin, "On Public and Private Blockchains," Aug. 2015. [Online]. Available: https://blog.ethereum.org/2015/08/07/on-public-and-privateblockchains_[Accessed: 8 May 2018].

[26] H. Vranken, "Sustainability of Bitcoin and Blockchains," Current Opinion in Environmental Sustainability, vol. 28, pp. 1-9, 2017. http://doi.org/10.1016/j.cosust.2017.04.011

[27] L. S. Sankar, M. Sindhu, and M. Sethumadhavan, "Survey of Consensus Protocols on Blockchain Applications," in 4th IEEE International Conference on Advanced Computing and Communication Systems. https://doi.org/10.1109/ICACCS.2017.8014672 
[28] K. Croman, C. Decker, I. Eyal, A. Gencer, R. Wattenhofer et al. "On Scaling Decentralized Blockchains," in International Financial Cryptography Association: FC 2016 Workshops, 2016, LNCS 9604, pp. 106-125. Available: http://fc16.ifca.ai/bitcoin/papers/CDE+16.pdf [Accessed: 8 May 2018].

[29] S. Azouvi, M. Maller, and S. Meiklejohn, "Egalitarian Society or Benevolent Dictatorship: The State of Cryptocurrency Governance," in 22nd International Conference on Financial Cryptography and Data Security, March 2018. Available: https://fc18.ifca.ai/bitcoin/papers/ bitcoin18-final13.pdf_[Accessed: 8 May 2018].

[30] F. Hofmann, W. Simone, E. Ron, and M. Böhmecke-Schwafert, "The Immutability Concept Of Blockchains And Benefits Of Early Standardization," in ITU Kaleidoscope: Challenges for a Data-Driven Society, Nov. 2017. https://doi.org/10.23919/ITU-WT.2017.8247004

[31] N. Alexopoulos, J. Daubert, M. Muhlhauser, and S. M. Habib, "Beyond the Hype: On Using Blockchains in Trust Management for Authentication," in 16th IEEE International Conference on Trust, Security and Privacy in Computing and Communications, Aug. 2017, pp. 546-553.

https://doi.org/10.1109/Trustcom/BigDataSE/ICESS.2017.283

[32] V. Lemieux, Blockchain Technology for Record Keeping: Help or Hype?, vol. 2. University of British Columbia, 2016. Available: https://www.researchgate.net/profile/Victoria_Lemieux_[Accessed: 8 May 2018].

[33] D. Anh, M. Zhang, B. C. Ooi, and G. Chen, "Untangling Blockchain: A Data Processing View of Blockchain Systems," in IEEE Transactions on Knowledge and Data Engineering, Jan. 2018. https://doi.org/10.1109/TKDE.2017.2781227

[34] A. Miller, "Securing Blockchains," IC3 Retreat, May 2016. Available: https://goo.gl/HuSrU8_Accessed: 8 May 2018].

[35] InterPARES Trust Terminology Project: Key Blockchain Terms and Definitions, 2017. [Online]. Available: http://arstweb.clayton.edu/interlex/blockchain/[Accessed: 8 May 2018].

[36] F. Dai, Y. Shi, N. Meng, L. Wei, and Z. Ye, "From Bitcoin to Cybersecurity: Comparative Study of Blockchain Application and Security Issues," in IEEE 4th International Conference on Systems and Informatics, Nov. 2017, pp. 975-979. https://doi.org/10.1109/ICSAI.2017.8248427

[37] K. Fanning and D. B. Centers, "Blockchain and Its Coming Impact on Financial Services," J. Corp. Account. Finance, vol. 27, no. 5, 2016. http://doi.org/10.1002/jcaf.22179

[38] G. Magyar, "Blockchain: Solving the Privacy and Research Availability Tradeoff for EHR Data," in IEEE 30th Jubilee Neumann Colloquium, 2017. https://doi.org/10.1109/NC.2017.8263269

[39] H. Wang, K. Chen, and D. Xu, "A Maturity Model for Blockchain adoption," Financial Innovation, vol. 2, no. 1, 2016. https://doi.org/10.1186/s40854-016-0031-z

[40] J. Yli-Huumo, D. Ko, S. Choi, S. Park, and K. Smolander, "Where is Current Research on Blockchain Technology? - A Systematic Review," PLOS ONE, vol. 11, no. 10, 2016. http://doi.org/10.1371/journal.pone.0163477

[41] F. Lamberti, V. Gatteschi, C. Demartini, C. Pranteda, and V. Santamaria, "Blockchain or not Blockchain, That is the Question of the Insurance and Other Sectors," IT Professional, June 2017. https://doi.org/10.1109/MITP.2017.265110355

[42] L. M. Bech and R. Garratt, "Central Bank Cryptocurrencies," BIS Quarterly Review, Sep. 2017. [Online]. Available: https://www.bis.org/publ/qtrpdf/r_qt1709f.htm_[Accessed: 8 May 2018].

[43] T. Bocek, B. Rodrigues, T. Straser, and B. Stiller, "Blockchains Everywhere - A Use-Case of Blockchains in the Pharma Supply-Chain," in IFIP/IEEE Symposium on Integrated Network and Service Management, May 2017, pp. 772-777. https://doi.org/10.23919/INM.2017.7987376

[44] Z. Chen and Y. Zhu, "Personal Archive Service System Using Blockchain Technology: Case Study, Promising and Challenging," in IEEE International Conference on AI \& Mobile Services, June 2017, pp. 93-99. https://doi.org/10.1109/AIMS.2017.31

[45] C. Huls, "A Scenario Planning for Interbank Payments and Decentralized Ledger Platforms," Master thesis, University of Twente, Utrecht, 2015. Available: http://essay.utwente.nl/66966/1/Huls_MA_EWI.pdf_ [Accessed: 8 May 2018].

[46] M. Izmaylov, P. Anderson, A. Lemble, and J. Vysoky, A Practical Application of Blockchain for the Travel Industry. 2018. Available: https://windingtree.com/\#downloads_[Accessed: 8 May 2018].
[47] V. Lemieux, "Blockchain and Distributed Ledgers as Trusted Recordkeeping Systems: An Archival Theoretic Evaluation Framework," in IEEE Future Technologies Conference, June 2017. Available: https://www.researchgate.net/profile/Victoria_Lemieux_[Accessed: 8 May 2018].

[48] R. Lewis, "30 Things You can do With the Blockchain," 2016. [Online]. Available: https://medium.com/yope-chain/30-things-you-can-do-with-ablockchain-b23b2ab39664_[Accessed: 8 May 2018].

[49] S. Porru, A. Pinna, M. Marchesi, and R. Tonelli, "Blockchain-Oriented Software Engineering: Challenges and New Directions," in IEEE 39th International Conference on Software Engineering Companion, May 2017, pp. 169-171. https://doi.org/10.1109/ICSE-C.2017.142

[50] A. Tandulwadikar, Blockchain in Banking: A Measured Approach, Cognizant Reports. April 2016. Available: https://www.cognizant.com/whitepapers/Blockchain-in-Banking-AMeasured-Approach-codex1809.pdf[Accessed: 8 May 2018].

[51] L. S. Sankar, M. Sindhu, and M. Sethumadhavan, "Survey of Consensus Protocols on Blockchain Applications," in 4th IEEE International Conference on Advanced Computing and Communication Systems, 2017. https://doi.org/10.1109/ICACCS.2017.8014672

[52] J. Seppälä, "The Role of Trust in Understanding the Effects of Blockchain on Business Models," Master thesis, Aalto University, Helsinki, Finland, 2016. Available: https://aaltodoc.aalto.fi/bitstream/handle/123456789/23302/master_Sepp älä_Jane_2016.pdf? sequence $=1$ [Accessed: 8 May 2018].

[53] V. Lemieux, "Typology of Blockchain Recordkeeping Solutions and Some Reflections on their Implications for the Future of Archival Preservation," in IEEE International Conference on Big Data, Dec. 2017. https://doi.org/10.1109/BigData.2017.8258180

[54] S. K. Lo, X. Xu, Y. K. Chiam, and Q. Lu, "Evaluating Suitability of Applying Blockchain," in IEEE 22nd International Conference on Engineering Complex Computer Systems, 2017, pp. 158-161. https://doi.org/10.1109/ICECCS.2017.26

[55] M. E. Peck, "Blockchain world - Do You Need a Blockchain? This Chart Will Tell You if the Technology Can Solve Your Problem," IEEE Spectrum, vol. 54, issue 10, pp. 38-60, 2017. https://doi.org/10.1109/MSPEC.2017.8048838

[56] D. Šalehar, "Pattern Based Evaluation of Blockchain Technology as a catalyst for Business Model Innovation," Master thesis, Delft University of Technology, Netherlands, 2017. Available: http://repository.tudelft.nl/ [Accessed: 8 May 2018].

[57] T. D. Smith, "The Blockchain Litmus Test," in IEEE International Conference on Big Data, Dec. 2017, pp. 2217-2226. https://doi.org/10.1109/BigData.2017.8258183

[58] Mercator Advisory Group Inc., Are Blockchain Solutions Ready? Three Blockchain Solutions Put to the Test. Oct. 2016. Available: https://www.co-opfs.org/media/Mercator-Blockchain-Whitepaper.pdf [Accessed: 8 May 2018].

[59] F. Glaser, "Pervasive Decentralisation of Digital Infrastructures: A Framework for Blockchain enabled System and Use Case Analysis," in Proc. 50th Hawaii International Conference on System Science, 2017, pp. 1543-1552. Available: http://hdl.handle.net/10125/41339_[Accessed: 8 May 2018].

[60] Ministry of Economy, Trade and Industry of Japan, Evaluation Forms for Blockchain-Based Systems. 2017. Available: http://www.meti.go.jp/english/press/2017/pdf/0329_004a.pdf_c

[61] L. Aniello, R. Baldoni, E. Gaetani, F. Lombardi, A. Margheri, and V. Sassone, "A Prototype Evaluation of a Tamper-Resistant High Performance Blockchain-Based Transaction Log for a Distributed Database," in IEEE 13th European Dependable Computing Conference, Sep. 2017, pp. 151-154. https://doi.org/10.1109/EDCC.2017.31

[62] A. Brinckman, D. Luc, J. Nabrzyski, G. L. Neidig, J. Neidig, T. A. Puckett, S. K. Radha, and I. J. Taylor, "A Comparative Evaluation of Blockchain Systems for Application Sharing Using Containers," in IEEE 13th International Conference on eScience, Oct. 2017, pp. 490-497. https://doi.org/10.1109/eScience.2017.80

[63] A. E. Gencer and E. G. Sirer, "Miniature World: Measuring and Evaluating Blockchains," Cornell University, 2016. [Online]. Available: http://hackingdistributed.com/2017/02/10/miniature-world/

[64] K. Stinchcombe, "Ten Years in, Nobody Has Come up With a Use for Blockchain,” Dec. 2017. [Online]. Available: https://hackernoon.com/ten-years-in-nobody-has-come-up-with-a-usecase-for-blockchain-ee98c180100[Accessed: 8 May 2018]. 
[65] J. Ovenden, "Why Blockchain Hype Must End," Innovation Enterprise, 2018. Available:

https://channels.theinnovationenterprise.com/articles/why-blockchainhype-must-end [Accessed: 8 May 2018].

[66] D. Yaga, P. Mell, N. Roby, and K. Scarfone, Draft NISTIR 8202: Blockchain Tecnology Overview. NIST, 2018. Available: https://csrc.nist.gov/publications/detail/nistir/8202/draft_[Accessed: 8 May 2018].

[67] J. Stolfi, Review of NISTIR 8202. Institute of Computing at State University of Campinas, Brazil, 2018. Available:

https://www.ic.unicamp.br/ stolfi/temp/nist-report-review.pdf [Accessed: 8 May 2018].

[68] D. Gerard, Attack of the 50 Foot Blockchain: Bitcoin, Blockchain, Ethereum \& Smart Contracts. CreateSpace Independent Publishing Platform, 2017, $182 \mathrm{p}$.

Kaspars Zīle is a Master student majoring in Business Informatics at Riga Technical University. He has earned a Bachelor's degree in Business Management at Riga Technical University. His professional experience includes several years of leading different IT projects in banking and telecommunications and developing different e-commerce related products.

E-mail:kaspars.zile@outlook.com

Renāte Strazdina currently is the Head of Microsoft Latvia and Leading Researcher/Lecturer at Riga Technical University. Her previous experience includes high level positions at Ernst \& Young and PricewaterhouseCoopers. She earned her Doctoral degree at Riga Technical University in 2006.

E-mail: Renate.Strazdina@rtu.lv 